\title{
Allelopathic potential of Cassia fistula against Lactuca sativa, Setaria italica and Pennisetum americanum
}

\author{
Zahir Muhammad ${ }^{*}$, Naila Inayat ${ }^{1}$, Rehmanullah $^{1}$, Wisal Muhammad \\ $\mathrm{Khan}^{2}$ and Abdul Majeed ${ }^{3}$ \\ 1. Department of Botany, University of Peshawar, Peshawar, Khyber Pakhtunkhwa-Pakistan \\ 2. Department of Botany, Islamia College Peshawar, Khyber Pakhtunkhwa-Pakistan \\ 3. Department of Botany, Govt. Degree College Naguman, Peshawar, Khyber Pakhtunkhwa-Pakistan \\ *Corresponding author's email: kzahirmuhammad@yahoo.com
}

Citation

Zahir Muhammad, Naila Inayat, Rehmanullah, Wisal Muhammad Khan and Abdul Majeed. Allelopathic potential of Cassia fistula against Lactuca sativa, Setaria italica and Pennisetum americanum. Pure and Applied Biology. Vol. 8, Issue 1, pp187-198. http://dx.doi.org/10.19045/bspab.2018.700176

\begin{tabular}{llll}
\hline \hline Received: 04/05/2018 & Revised: 06/10/2018 & Accepted: 18/10/2018 & Online First: 25/10/2018 \\
\hline
\end{tabular}

\section{Abstract}

Plants during different phases of growth produce secondary metabolites which have multipurpose roles. One of the aspired roles associated with the production of such compounds is their active involvement in allelopathy -a phenomenon of the chemical interactions of plants and/or microorganisms. Investigation of the allelopathic properties of different plants is useful in agriculture as it would lead to the proper cultivation of crops under field conditions with respect to allelopathic plants. This study was aimed at exploring the allelopathic effects of Cassia fistula aqueous extracts obtained from leaves, bark, and fruit on germination and growth of Lactuca sativa, Setaria italica and Pennisetum americanum. Results demonstrated that aqueous extract from leaves, bark, and fruit of $C$. fistula had differential toxicity against the Lactuca sativa, Setaria italica and Pennisetum americanum. Phytotoxicity of extracts depended upon the concentration soaking duration. It was observed that 24-hour extracts were more inhibitory. Leaf extracts exhibited more toxicity than bark and fruit. Lactuca sativa was found more sensitive to the applied allelopathic stress of $C$. fistula than Setaria italica and Pennisetum americanum. Based on our results, it is suggested that Cassia fistula has strong allelopathic potential and may serve as a potential candidate for using its allelopathic potentials in weed control strategies.

Keywords: Allelochemicals; Allelopathy; Cassia fistula; Phytotoxicity; Weed control strategies

\section{Introduction}

The term "allelopathy" infers to the communications between plants and/or microbes which might lead to either stimulation or inhibition of growth, physiology, and development of plants and other organisms by the release of secondary molecules termed as 'allelochemicals' [1]. In nature, different groups of plants like algae, lichens, crops, and weeds have well established allelopathic properties which are thought to be an evolutionary mechanism adopted by plants to survive in the given environment and to determine their success over susceptible plant species [2, 3]. Diverse phytotoxic substances causing germination and/or growth inhibitions have been isolated from several plants and their tissues, which 
are known as allelochemicals -secondary plant metabolites which interact with other plants in diverse mechanisms to either suppress or promote their growth, depending on the types of communicating plants, the environment and nature of allelopathic substances $[\mathbf{1 , 4}, \mathbf{5}]$.

The allelopathic effects of one plant on the other are generally discerning and may greatly vary since the production and nature of biological activities of secondary compounds in different plants and their tissues are variable $[6,7]$. Leaves are generally perceived to possess most of the potent sources of allelochemicals, albeit other plant tissues are also known to produce allelopathic chemicals with differential phyto-stimulatory or phyto-inhibitory influences. Cassia fistula, commonly known as Indian laburnum, is an important tree with well-known antimicrobial, antifungal and antioxidant activities [8, 9] however, very little work has been done on the allelopathic effect of $C$. fistula on another crop so far. Therefore this experiment was conducted with the aim of exploring the allelopathic effects of leaf, bark and fruit extracts of $C$. fistula on Lactuca sativa, Setaria italica and Pennisetum americanum.

\section{Materials and methods}

Cassia fistula was selected as the donor plant and the receptor plants selected were Lactuca sativa, Setaria italica and Pennisetum americanum. The aqueous extracts were prepared from leaf, bark, and fruit of the donor plant. A 5-10g of leaves, bark, and fruit from donor plant were soaked in $100 \mathrm{ml}$ of distil water for 24 and $48 \mathrm{hrs}$ soaking duration and filtered through Whatman filter paper and stored for seed treatment experiments. Extracts preparation and seedling bioassay was performed following the method of Muhammad et al. [10].

\section{Germination and growth records}

The germination test was carried out in sterile Petri dishes of $9 \mathrm{~cm}$ in size placing a
Whatman filter paper on Petri dishes. Ten seeds each of Lactuca sativa, Setaria italica and Pennisetum americanum were grown in Petri dishes containing double folded Wattman filter paper. Each Petri dish was provided with $10 \mathrm{ml}$ of respective extracts i.e., $5 \mathrm{~g}$ and $10 \mathrm{~g}$ extract of leaf, bark, and fruit. Distilled water was used as a control. Each treatment was replicated three times and the experiment was arranged in a completely randomized design (CRD). Petri dishes were kept in the RCB design incubator for 72 hours at $25^{\circ} \mathrm{C}$. Data for germination percentage, radical and hypocotyl length was recorded after $72 \mathrm{hrs}$.

\section{Statistical analysis}

Data were analyzed by analysis of variance (ANOVA), using the MstatC software and difference were considered statistically significant at $\mathrm{p} \leq 0.05$.

\section{Results}

\section{Effect of leaves extract}

Germination (\%)

Results demonstrated significant $(p \leq 0.05)$ differences for germination (\%) between concentrations, varieties, and soaking durations. Furthermore, the interactive effect of varieties (V), concentrations (Cs) and soaking durations $(\mathrm{H})$ i.e. $\mathrm{V} \times \mathrm{Cs}$ and $\mathrm{Hx} \mathrm{V} x$ $\mathrm{Cs}$ were also significant. However, $\mathrm{H} \mathrm{x} \mathrm{V}$ and $\mathrm{H} \times \mathrm{Cs}$ interaction were non-significant. Extracts from leaves of Cassia fistula were phytotoxic to the germination $\%$ of test species i.e. Lactuca sativa, Setaria italica and Pennisetum americanum. However, the inhibition magnitude varied between them. Inhibition of the extract was concentration dependent. Maximum germination \% was recorded in control (78\%), followed by $5 \mathrm{~g}$ conc. (49.66\%) and $10 \mathrm{~g}$ conc. (39.66). Varietal response to different concentration showed maximum germination in $P$. americanum (71\%), followed by $S$. italica $(65.33 \%)$ and L. sativa (31\%). Effect of soaking duration obtained from leaves after 
$24 \mathrm{~h}(50.89 \%)$ was more inhibitory than $48 \mathrm{~h}$ extracts (60.67) (Table 1).

Table 1. Mean squares of the ANOVA for germination (\%), radical and hypocotyl growth (mm) of leaves aqueous extract

\begin{tabular}{|c|c|c|c|c|}
\hline Sources & d. $\mathbf{f}$ & Germination (\%) & $\begin{array}{c}\text { Hypocotyl growth } \\
(\mathrm{mm})\end{array}$ & $\begin{array}{c}\text { Radical growth } \\
(\mathbf{m m})\end{array}$ \\
\hline Varieties (V) & 2 & $14054^{*}$ & $53.59^{*}$ & $51.08^{*}$ \\
\hline Concentrations $(\mathrm{C})$ & 2 & $11861^{*}$ & $10.99^{*}$ & $21.59^{*}$ \\
\hline $\mathrm{V} \times \mathrm{C}$ & 4 & $4011^{*}$ & $5.43^{*}$ & $4.47^{*}$ \\
\hline Hours $(\mathrm{H})$ & 1 & $2151^{*}$ & $17.68^{\mathrm{NS}}$ & $2.61^{*}$ \\
\hline $\mathrm{H}^{*} \mathrm{~V}$ & 2 & $408^{\mathrm{NS}}$ & $16.91^{*}$ & $1.30^{*}$ \\
\hline $\mathrm{H}^{*} \mathrm{C}$ & 2 & $474^{\mathrm{NS}}$ & $7.72^{*}$ & $4.08^{*}$ \\
\hline $\mathrm{H}^{*} \mathrm{~V}^{*} \mathrm{C}$ & 4 & $1841^{*}$ & $8.69^{*}$ & $1.70^{*}$ \\
\hline Error & 72 & 214 & 0.49 & 0.38 \\
\hline \multicolumn{2}{|c|}{ Coefficient of Variation (\%) } & $26.25 \%$ & $40.37 \%$ & $37.93 \%$ \\
\hline
\end{tabular}

d. $\mathrm{f}=$ degree of freedom, $*$ = significant and $\mathrm{NS}=$ non-significant

\section{Root elongation}

Analysis of variance (ANOVA) exhibited significant $(p \leq 0.05)$ differences among means value for radical length i.e. $\mathrm{V}, \mathrm{Cs}, \mathrm{H}$ and their interactive effect $\mathrm{V} \times \mathrm{Cs}, \mathrm{H} \times \mathrm{V}, \mathrm{H} \times$ $\mathrm{Cs}$ and $\mathrm{H} \times \mathrm{V} \times \mathrm{Cs}$. Radical lengths of $L$. sativa, $S$. italica and $P$. ameracanum were found to be greatly inhibited with the increase in the concentration of the extracts. The maximum radical length was recorded in control $(2.3 \mathrm{~mm})$, followed by $5 \mathrm{~g}$ conc. $(1.88$ $\mathrm{mm}$ ) and $10 \mathrm{~g}$ conc. (0.66) with a gradual decrease. Varietal response elucidated maximum radical length in $P$. ameracanum $3.08 \mathrm{~mm}$ ), followed by $S$. italica (1.19) and $L$. sativa $(0.58 \mathrm{~mm})$. Soaking duration means revealed that $24 \mathrm{~h}(1.45 \mathrm{~mm})$ duration was inhibitory than $48 \mathrm{~h}$ extracts $(1.79 \mathrm{~mm})$ (Table 2).

\section{Shoot elongation}

ANOVA for the hypocotyl length showed a significant difference for varieties, concentrations and soaking duration, and their interactive effect $\mathrm{V} \times \mathrm{Cs}, \mathrm{H} \times \mathrm{V}, \mathrm{H} \times \mathrm{Cs}$ and $\mathrm{H} x \mathrm{~V} \times \mathrm{Cs} .(p \leq 0.05)$. However, soaking duration means were non-significant (Table 3). Concentration means specified that radical length was maximum in control $(2.32 \mathrm{~mm})$ which decreased significantly in a dose-dependent manner to a minimum of $(1.63 \mathrm{~mm})$ at $5 \mathrm{~g}$ conc. and $10 \mathrm{~g}$ conc. $(1.09 \mathrm{~mm})$. According to varietal response maximum radical length was noted in $P$. ameracnum $(2.98 \mathrm{~mm})$, followed by $S$. italica $(1.37 \mathrm{~mm})$ and L. sativa $(0.69 \mathrm{~mm})$. Soaking Durant means reveals that $24 \mathrm{~h}(1.67 \mathrm{~mm})$ duration was more inhibitory than $48 \mathrm{~h}$ extracts $(1.69 \mathrm{~mm})$ with non-significant means (Table 4). 
Table 2. Mean squares of the ANOVA for germination (\%), radical and hypocotyl growth (mm) of bark aqueous extract

\begin{tabular}{|c|c|c|c|c|}
\hline Sources & d. $f$ & Germination $(\%)$ & $\begin{array}{c}\text { Hypocotyl Growth } \\
(\mathrm{mm})\end{array}$ & $\begin{array}{c}\text { Radical } \\
\text { Growth }(\mathbf{m m})\end{array}$ \\
\hline Varieties (V) & 2 & $12654^{*}$ & $90.61^{*}$ & $140.7^{*}$ \\
\hline Concentrations (C) & 2 & $3217^{*}$ & $0.09^{\mathrm{NS}}$ & $1.55^{\mathrm{NS}}$ \\
\hline $\mathrm{V} \times \mathrm{C}$ & 4 & $3054^{*}$ & $8.18^{*}$ & $10.18^{*}$ \\
\hline Hours $(\mathrm{H})$ & 1 & $1137^{*}$ & $0.39^{\mathrm{NS}}$ & $6.66^{*}$ \\
\hline $\mathrm{H}^{*} \mathrm{~V}$ & 2 & $201^{\text {NS }}$ & $4.80^{*}$ & $5.27^{*}$ \\
\hline $\mathrm{H}^{*} \mathrm{C}$ & 2 & $271^{\text {NS }}$ & $2.12^{*}$ & $17.15^{*}$ \\
\hline $\mathrm{H}^{*} \mathrm{~V} * \mathrm{C}$ & 4 & $1194^{*}$ & $1.90^{*}$ & $9.43^{*}$ \\
\hline Error & 72 & 141 & 0.24 & 0.59 \\
\hline \multicolumn{2}{|c|}{ Coefficient of Variation (\%) } & $17.08 \%$ & $20.85 \%$ & $32.85 \%$ \\
\hline
\end{tabular}

d. $\mathrm{f}=$ degree of freedom, $*=$ significant and $\mathrm{NS}=$ non-significant

Table 3. Mean squares of the ANOVA for germination (\%), radical and hypocotyl growth (mm) of fruit aqueous extract

\begin{tabular}{|c|c|c|c|c|}
\hline Sources & d.f & Germination (\%) & $\begin{array}{c}\text { Hypocotyl growth } \\
(\mathbf{m m})\end{array}$ & Radical growth (mm) \\
\hline Varieties (V) & 2 & $9910^{*}$ & $57.19^{*}$ & $53.59^{*}$ \\
\hline Concentrations (C) & 2 & $2773^{*}$ & $2.44^{*}$ & $10.99^{*}$ \\
\hline $\mathrm{V} \times \mathrm{C}$ & 4 & $873^{*}$ & $2.55^{*}$ & $5.43^{*}$ \\
\hline Hours $(\mathrm{H})$ & 1 & $111^{\mathrm{NS}}$ & $2.61^{*}$ & $17.68^{*}$ \\
\hline $\mathrm{H}^{*} \mathrm{~V}$ & 2 & $67^{\mathrm{NS}}$ & $7.44^{*}$ & $16.91^{*}$ \\
\hline $\mathrm{H}^{*} \mathrm{C}$ & 2 & $2364^{*}$ & $0.45^{\mathrm{NS}}$ & $7.73^{*}$ \\
\hline $\mathrm{H}^{*} \mathrm{~V}^{*} \mathrm{C}$ & 4 & $1311^{*}$ & $2.09^{*}$ & $8.69^{*}$ \\
\hline Error & 72 & 154 & 0.29 & 0.49 \\
\hline \multicolumn{2}{|c|}{ Coefficient of Variation (\%) } & $17.75 \%$ & $26.73 \%$ & $40.37 \%$ \\
\hline
\end{tabular}

d. $\mathrm{f}=$ degree of freedom, $*=$ significant and $\mathrm{NS}=$ non-significant 
Table 4. Effects of aqueous extracts from leaves of Cassia fistula on the final germination percentage $(\%)$ of various test plants

\begin{tabular}{|c|c|c|c|c|c|c|}
\hline \multirow{2}{*}{$\begin{array}{l}\text { Soaking } \\
\text { duration }\end{array}$} & \multirow{2}{*}{ Treat. } & \multicolumn{3}{|c|}{ Varieties } & \multirow{2}{*}{$\begin{array}{c}\text { Means } \\
\text { Hrs*Conc. }\end{array}$} & \multirow{2}{*}{$\begin{array}{c}\text { Means } \\
\text { Var.*Conc. }\end{array}$} \\
\hline & & Lactuca & Setaria & Penesitum & & \\
\hline \multirow{3}{*}{$24 \mathrm{hrs}$} & Con & $60.00^{\mathrm{DEF}}$ & $88.00^{\mathbf{A B}}$ & $66.00^{\mathrm{CDE}}$ & 71.33 & $78.00^{\mathrm{A}}$ \\
\hline & $5 g$ & $30.00^{\mathbf{G}}$ & $44.00^{\mathrm{FG}}$ & $74.00^{\mathrm{BCD}}$ & 49.33 & $15.00^{\mathbf{D}}$ \\
\hline & $10 \mathrm{~g}$ & $0.0^{\mathbf{H}}$ & $48.00^{\mathbf{E F G}}$ & $48.00^{\mathbf{E F G}}$ & 32.00 & $0.0^{\mathbf{E}}$ \\
\hline \multicolumn{2}{|c|}{ Means hrs*var. } & $30.00^{\mathbf{D}}$ & $60.00^{\mathrm{C}}$ & $62.67^{\mathrm{BC}}$ & $50.89^{\mathbf{B}}$ & $83.00^{\mathrm{A}}$ \\
\hline \multirow{3}{*}{$48 \mathrm{hrs}$} & Con & $96.00^{\mathbf{A}}$ & $78.00^{\mathrm{ABCD}}$ & $80.00^{\mathrm{ABC}}$ & 84.67 & $58.00^{\mathrm{C}}$ \\
\hline & $5 \mathrm{~g}$ & $0.0^{\mathrm{H}}$ & $72.00^{\mathbf{B C D}}$ & $78.00^{\mathrm{ABCD}}$ & 50.00 & $55.00^{\mathrm{C}}$ \\
\hline & $10 \mathrm{~g}$ & $0.0^{\mathrm{H}}$ & $62.00^{\mathrm{CDEF}}$ & $80.00^{\mathrm{ABC}}$ & 47.33 & $73.00^{\mathrm{AB}}$ \\
\hline \multicolumn{2}{|c|}{ Means hrs*var. } & $32.00^{\mathrm{D}}$ & $70.66^{\mathrm{AB}}$ & $79.33^{\mathrm{A}}$ & $60.67^{\mathrm{A}}$ & $76.00^{\mathrm{AB}}$ \\
\hline \multicolumn{2}{|c|}{ Means varieties } & $31.00^{\mathbf{B}}$ & $65.33^{\mathrm{A}}$ & $71.00^{\mathbf{A}}$ & & $64.00^{\mathrm{BC}}$ \\
\hline \multicolumn{2}{|c|}{ Means concentration } & $78.00^{\mathrm{A}}$ & $49.66^{\mathbf{B}}$ & $39.66^{\mathrm{C}}$ & & \\
\hline
\end{tabular}

LSD value for Hours $=10.66$, Varieties $=7.537$, Concentration $=7.54$, Varieties $*$ Concentrations $=13.06$, Hours*Variety $=10.66$ and Hours*Variety*Concentration $=18.46$ interactions at $\alpha 5 \%$. Mean values which are not followed by the same letter are statistically significant at $\mathrm{p}<0.05$

Effect of bark extract

\section{Germination (\%)}

ANOVA table indicated that varietal, concentration and soaking duration effects and the interaction between $\mathrm{V} \times \mathrm{Cs}$ and $\mathrm{H} \times \mathrm{V}$ $\mathrm{x}$ Cs. were significant $(p \leq 0.05)$. However, $\mathrm{H} \times \mathrm{V}, \mathrm{H} \times \mathrm{Cs}$ means were non-significant (Table 5). Germination bioassay demonstrated that the three test varieties differed in their response to the applied extracts; showing decline at increasing concentration of extracts. In bark aqueous extract treatments, maximum germination percentage $(78 \%)$ was recorded in control, followed by $5 \mathrm{~g}(72.66 \%)$ and $10 \mathrm{~g}(58 \%)$. Varietal means bestowed maximum germination in $P$. ameracanum (85\%), followed by S. italica $(77 \%)$ and $L$. sativa (46.33\%). Soaking duration means shows maximum germination inhibition $24 \mathrm{~h}$ $(58.66 \%)$ which was more inhibitory than $48 \mathrm{~h}$ extracts $(66 \%)$ (Table 6)

\section{Root elongation}

ANOVA table revealed that the response of varieties, concentration and soaking durations and interaction between $\mathrm{V} \times \mathrm{Cs}, \mathrm{H} x$ $\mathrm{V}, \mathrm{H} \times \mathrm{Cs}$ and $\mathrm{H} \times \mathrm{V}$ x Cs. were significant $(p \leq 0.05)$. Nevertheless, Concentrations means were non-significant (Table 5). Means table for radical length revealed that all the extract concentrations were non-significant, however, maximum radical length $(2.61 \mathrm{~mm})$ was observed at $5 \mathrm{~g}$ conc. followed by control $(2.30 \mathrm{~mm})$ and $10 \mathrm{~g}$ (2.16). Varieties mean elucidated maximum radical length in $P$. ameracanum $(4.86 \mathrm{~mm})$, followed by $S$. italica (1.19) and L. sativa (1.03mm). Soaking duration means showed maximum radical length in $24 \mathrm{~h}(2.63 \mathrm{~mm})$ and minimum radical length in $48 \mathrm{~h}(2.08 \mathrm{~mm})$ respectively (Table 7).

\section{Shoot elongation}

ANOVA for the hypocotyl length showed a significant difference for $\mathrm{V}$ and their interactive effect $\mathrm{V} \times \mathrm{Cs}, \mathrm{H} \times \mathrm{V}, \mathrm{H} \times \mathrm{Cs}$ and $\mathrm{H}$ $\mathrm{x} \mathrm{V} \times \mathrm{Cs}(p \leq 0.05)$. However, soaking duration and concentration means were nonsignificant (Table 5). Concentration means indicated that plumule length was maximum in $10 \mathrm{~g}(2.38 \mathrm{~mm})$ followed by control $(1.63 \mathrm{~mm})$ and $10 \mathrm{~g}$ conc. (2.26mm). According to varietal means, maximum radical length was noted in $P$. americanum $(4.33 \mathrm{~mm})$, followed by $S$. italica $(1.51 \mathrm{~mm})$ 
and L. sativa $(1.17 \mathrm{~mm})$. Soaking duration means reveals that $24 \mathrm{~h}(2.40 \mathrm{~mm})$ duration was inhibitorier than $48 \mathrm{~h}$ extracts $(2.27 \mathrm{~mm})$ with non-significant means (Table 8).

Table 5. Effects of aqueous extracts from leaves of Cassia fistula on the radical length (mm) of various test plants

\begin{tabular}{|c|c|c|c|c|c|c|}
\hline \multirow{2}{*}{$\begin{array}{l}\text { Soaking } \\
\text { duration }\end{array}$} & \multirow{2}{*}{ Treat. } & \multicolumn{3}{|c|}{ Varieties } & \multirow{2}{*}{$\begin{array}{c}\text { Means } \\
\text { Hrs*Conc. }\end{array}$} & \multirow{2}{*}{$\begin{array}{c}\text { Means } \\
\text { Var.*Conc }\end{array}$} \\
\hline & & Lactuca & Setaria & Penesitum & & \\
\hline \multirow{3}{*}{$24 \mathrm{hrs}$} & Con & $1.56^{\mathrm{CD}}$ & $1.83^{\mathrm{CD}}$ & $3.29^{\mathrm{B}}$ & $2.23^{\mathrm{A}}$ & $1.54^{\mathrm{CD}}$ \\
\hline & $5 \mathrm{~g}$ & $0.36^{\mathrm{F}}$ & $0.22^{\mathbf{F}}$ & $3.32^{\mathrm{B}}$ & $1.30^{\mathbf{B}}$ & $0.18^{\mathbf{E}}$ \\
\hline & $10 \mathrm{~g}$ & $0^{\mathbf{F}}$ & $0.558^{\mathrm{EF}}$ & $1.88^{\mathrm{CD}}$ & $0.82^{\mathrm{C}}$ & $0^{\mathbf{E}}$ \\
\hline \multicolumn{2}{|c|}{ Means hrs*var. } & $0.64^{\mathrm{D}}$ & $0.87^{\mathbf{D}}$ & $2.83^{\mathrm{B}}$ & 1.45 & $1.89^{\mathrm{C}}$ \\
\hline \multirow{3}{*}{$48 \mathrm{hrs}$} & Con & $1.53^{\mathrm{D}}$ & $1.96^{\mathrm{CD}}$ & $3.65^{\mathrm{B}}$ & $2.38^{\mathbf{A}}$ & $1.27^{\mathrm{D}}$ \\
\hline & $5 g$ & $0^{\mathbf{F}}$ & $2.33^{\mathrm{C}}$ & $5.04^{\mathrm{A}}$ & $2.46^{\mathbf{A}}$ & $0.41^{\mathrm{E}}$ \\
\hline & $10 \mathrm{~g}$ & $0^{\mathbf{F}}$ & $0.26^{\mathbf{F}}$ & $1.31^{\mathrm{DE}}$ & $0.52^{\mathrm{C}}$ & $3.47^{\mathrm{B}}$ \\
\hline \multicolumn{2}{|c|}{ Means hrs*var. } & $0.51^{\mathrm{D}}$ & $1.52^{\mathrm{C}}$ & $3.33^{\mathrm{A}}$ & 1.79 & $4.18^{\mathrm{A}}$ \\
\hline \multicolumn{2}{|c|}{ Means varieties } & 0.58 & 1.19 & 3.08 & & $1.59^{\mathrm{CD}}$ \\
\hline \multicolumn{2}{|c|}{ Means concentration } & $2.3^{\mathrm{A}}$ & $1.88^{\mathbf{B}}$ & $0.66^{\mathrm{C}}$ & & \\
\hline
\end{tabular}

LSD value for Hours $=$, Varieties $=0.32$, Concentration $=0.32$, Hours*Varieties $=0.44$, Hours*Concentration $=0.45$, Varieties $*$ Concentrations $=0.55$ and Hours*Variety $*$ Concentration $=0.77$ interactions at $\alpha 5 \%$. Mean values which are not followed by the same letter are statistically significant at $\mathrm{p}<0.05$

Table 6. Effects of aqueous extracts from leaves of Cassia fistula on the hypocotyl length (mm) of various test plants

\begin{tabular}{|c|c|c|c|c|c|c|}
\hline \multirow{2}{*}{$\begin{array}{l}\text { Soaking } \\
\text { duration }\end{array}$} & \multirow{2}{*}{ Treat. } & \multicolumn{3}{|c|}{ Varieties } & \multirow{2}{*}{$\begin{array}{c}\text { Means } \\
\text { Hrs*Conc. }\end{array}$} & \multirow{2}{*}{$\begin{array}{c}\text { Means } \\
\text { Var.*Conc }\end{array}$} \\
\hline & & Lactuca & Setaria & Penesitum & & \\
\hline \multirow{3}{*}{$24 \mathrm{hrs}$} & Con & $1.96^{\mathrm{CD}}$ & $1.86^{\mathrm{CD}}$ & $3.54^{\mathrm{A}}$ & $2.45^{\mathrm{A}}$ & $1.87^{\mathrm{B}}$ \\
\hline & $5 g$ & $0.39^{\mathbf{F}}$ & $0.33^{\mathbf{F}}$ & $3.25^{\mathrm{A}}$ & $1.32^{\mathrm{C}}$ & $0.19^{\mathrm{D}}$ \\
\hline & $10 \mathrm{~g}$ & $0.0^{\mathbf{F}}$ & $0.63^{\mathrm{EF}}$ & $3.06^{\mathrm{A}}$ & $1.23^{\mathrm{C}}$ & $0.0^{\mathbf{D}}$ \\
\hline \multicolumn{2}{|c|}{ Means hrs*var. } & $0.78^{\mathbf{D}}$ & $0.94^{\mathrm{D}}$ & $3.28^{\mathrm{A}}$ & 1.67 & $1.85^{\mathrm{B}}$ \\
\hline \multirow{3}{*}{$48 \mathrm{hrs}$} & Con & $1.78^{\mathrm{CD}}$ & $1.84^{\mathrm{CD}}$ & $2.92^{\mathrm{AB}}$ & $2.18^{\mathrm{AB}}$ & $1.27^{\mathrm{C}}$ \\
\hline & $5 g$ & $0.0^{\mathbf{F}}$ & $2.22^{\mathrm{BC}}$ & $3.56^{\mathrm{A}}$ & $1.93^{\mathrm{B}}$ & $0.97^{\mathrm{C}}$ \\
\hline & $10 \mathrm{~g}$ & $0.0^{\mathbf{F}}$ & $1.31^{\mathrm{DE}}$ & $1.57^{\mathrm{CD}}$ & $0.96^{\mathrm{C}}$ & $3.23^{\mathrm{A}}$ \\
\hline \multicolumn{2}{|c|}{ Means hrs*var. } & $0.59^{\mathrm{D}}$ & $1.79^{\mathrm{C}}$ & $2.68^{\mathrm{B}}$ & 1.69 & $3.40^{\mathrm{A}}$ \\
\hline \multicolumn{2}{|c|}{ Means varieties } & $0.69^{\mathrm{C}}$ & $1.37^{\mathbf{B}}$ & $2.98^{\mathrm{A}}$ & & $2.32^{\mathrm{B}}$ \\
\hline \multicolumn{2}{|c|}{ Means concentration } & $2.32^{\mathrm{A}}$ & $1.63^{\mathrm{B}}$ & $1.09^{\mathrm{C}}$ & & \\
\hline
\end{tabular}

LSD value for Varieties $=0.30$, Concentration $=0.30$, Hours*Varieties $=0.43$, Hours $*$ Concentration $=0.43$, Varieties ${ }^{*}$ Concentrations $=0.53$ and Hours*Variety*Concentration $=0.75$ interactions at $\alpha 5 \%$. Mean values which are not followed by the same letter are statistically significant at $p<0.05$ 
Table 7. Effects of aqueous extracts from bark of Cassia fistula on the final germination percentage $(\%)$ of various test plants

\begin{tabular}{|c|c|c|c|c|c|c|}
\hline \multirow{2}{*}{$\begin{array}{l}\text { Soaking } \\
\text { duration }\end{array}$} & \multirow{2}{*}{ Treat. } & \multicolumn{3}{|c|}{ Varieties } & \multirow{2}{*}{$\begin{array}{c}\text { Means } \\
\text { Hrs*Conc. }\end{array}$} & \multirow{2}{*}{$\begin{array}{c}\text { Means } \\
\text { Var.*Conc }\end{array}$} \\
\hline & & Lactuca & Setaria & Penesitum & & \\
\hline \multirow{3}{*}{$24 \mathrm{hrs}$} & Con & $60.00^{\mathbf{E F}}$ & $96.00^{\mathbf{A}}$ & $88.00^{\mathrm{AB}}$ & 81.33 & $69.00^{\mathrm{C}}$ \\
\hline & $5 \mathrm{~g}$ & $68.00^{\mathrm{CDE}}$ & $84.00^{\mathrm{AB}}$ & $86.00^{\mathrm{AB}}$ & 79.33 & $58.00^{\mathrm{D}}$ \\
\hline & $10 \mathrm{~g}$ & $24.00^{\mathbf{G}}$ & $68.00^{\mathrm{CDE}}$ & $84.00^{\mathbf{A B}}$ & 58.66 & $12.00^{\mathbf{E}}$ \\
\hline \multicolumn{2}{|c|}{ Means hrs*var. } & 50.67 & 82.67 & 86.00 & 73.11 & $81.00^{\mathbf{A B}}$ \\
\hline \multirow{3}{*}{$48 \mathrm{hrs}$} & Con & $78.00^{\mathrm{BCD}}$ & $66.00^{\mathrm{DE}}$ & $80.00^{\mathrm{BCD}}$ & 74.67 & $76.00^{\mathrm{BC}}$ \\
\hline & $5 \mathrm{~g}$ & $48.00^{\mathrm{F}}$ & $68.00^{\mathrm{CDE}}$ & $82.00^{\mathrm{ABC}}$ & 66.00 & $74.00^{\mathrm{BC}}$ \\
\hline & $10 \mathrm{~g}$ & $0.0^{\mathbf{H}}$ & $80.00^{\mathrm{BCD}}$ & $92.00^{\mathrm{AB}}$ & 57.33 & $84.00^{\mathrm{AB}}$ \\
\hline \multicolumn{2}{|c|}{ Means hrs*var. } & 42.00 & 71.33 & 84.67 & 66.00 & $84.00^{\mathrm{AB}}$ \\
\hline \multicolumn{2}{|c|}{ Means varieties } & $46.33^{C}$ & $77.00^{\mathbf{B}}$ & $85.00^{\mathrm{A}}$ & & $88.00^{\mathbf{A}}$ \\
\hline \multicolumn{2}{|c|}{ Means concentration } & $78.00^{\mathbf{A}}$ & $72.66^{\mathrm{A}}$ & $58.00^{\mathbf{B}}$ & & \\
\hline
\end{tabular}

LSD value for Hours $=10.66$, Varieties $=6.12$, Concentration $=6.12$, Varieties ${ }^{*}$ Concentrations $=10.59$ and Hours*Variety*Concentration $=14.98$ interactions at $\alpha 5 \%$. Mean values which are not followed by the same letter are statistically significant at $\mathrm{p}<0.05$

Table 8. Effects of aqueous extracts from bark of Cassia fistula on the radical length (mm) of various test plants

\begin{tabular}{|c|c|c|c|c|c|c|}
\hline \multirow{2}{*}{$\begin{array}{l}\text { Soaking } \\
\text { duration }\end{array}$} & \multirow{2}{*}{ Treat. } & \multicolumn{3}{|c|}{ Varieties } & \multirow{2}{*}{$\begin{array}{c}\text { Means } \\
\text { Hrs*Conc. }\end{array}$} & \multirow{2}{*}{$\begin{array}{c}\text { Means } \\
\text { Var.*Conc }\end{array}$} \\
\hline & & Lactuca & Setaria & Penesitum & & \\
\hline \multirow{3}{*}{$24 \mathrm{hrs}$} & Con & $1.56^{\mathrm{EFGH}}$ & $1.83^{\mathrm{EFG}}$ & $3.29^{\mathrm{D}}$ & $2.23^{\mathrm{C}}$ & $1.54^{\mathrm{DE}}$ \\
\hline & $5 \mathrm{~g}$ & $1.19^{\mathrm{FGHI}}$ & $0.58^{\mathrm{IJ}}$ & $5.31^{\mathrm{C}}$ & $2.36^{\mathrm{BC}}$ & $1.01^{\mathrm{EF}}$ \\
\hline & $10 \mathrm{~g}$ & $1.06^{\mathrm{GHI}}$ & $0.75^{\mathrm{HIJ}}$ & $8.08^{\mathrm{A}}$ & $3.29^{\mathrm{BC}}$ & $0.53^{\mathrm{F}}$ \\
\hline \multicolumn{2}{|c|}{ Means hrs*var. } & $1.27^{\mathrm{CD}}$ & $1.05^{\mathrm{D}}$ & $5.56^{\mathrm{A}}$ & $2.63^{\mathrm{A}}$ & $1.89^{\mathrm{D}}$ \\
\hline \multirow{3}{*}{$48 \mathrm{hrs}$} & Con & $1.52^{\text {EFGH }}$ & $1.96^{\mathrm{EF}}$ & $3.65^{\mathrm{D}}$ & $2.38^{\mathrm{BC}}$ & $0.95^{\mathrm{EF}}$ \\
\hline & $5 \mathrm{~g}$ & $0.82^{\mathrm{HIJ}}$ & $1.31^{\mathrm{FGHI}}$ & $6.42^{\mathrm{B}}$ & $2.85^{\mathrm{AB}}$ & $0.72^{\mathrm{F}}$ \\
\hline & $10 \mathrm{~g}$ & $0.0^{\mathrm{J}}$ & $0.68^{\mathrm{HIJ}}$ & $2.38^{\mathrm{E}}$ & $1.02^{\mathrm{D}}$ & $3.47^{\mathrm{C}}$ \\
\hline \multicolumn{2}{|c|}{ Means hrs*var. } & $0.78^{\mathbf{D}}$ & $1.32^{\mathrm{C}}$ & $4.15^{\mathrm{B}}$ & $2.08^{\mathrm{B}}$ & $5.87^{\mathrm{A}}$ \\
\hline \multicolumn{2}{|c|}{ Means varieties } & $1.03^{\mathrm{B}}$ & $1.19^{\mathbf{B}}$ & $4.86^{\mathrm{A}}$ & & $5.23^{\mathrm{B}}$ \\
\hline \multicolumn{2}{|c|}{ Means concentration } & $2.30^{\mathrm{AB}}$ & $2.61^{\mathrm{A}}$ & $2.16^{\mathrm{B}}$ & & \\
\hline
\end{tabular}

LSD value for Hours $=10.66$, Varieties $=0.39$, Hours*Varieties $=0.52$, Hours*Concentration $=0.52$, Varieties*Concentrations $=0.62$ and Hours*Variety*Concentration $=0.77$ interactions at $\alpha 5 \%$. Mean values which are not followed by the same letter are statistically significant at $\mathrm{p}<0.05$

Effect of fruit extract

Germination (\%)

Significant differences were observed for varieties, concentrations and interactions between them $(p \leq 0.05)$. However, soaking duration and interaction between concentration and soaking duration were non-significant (Table 9). According to concentration means, germination\% was maximum at control $(78 \%)$ followed by $5 \mathrm{~g}$ 
(72.67\%) and $10 \mathrm{~g}$ conc. (59.33\%). The varietal response exhibited maximum germination\% in P. americanum (84.67\%), followed by $S$. italica $(75.67 \%)$ and L. sativa $(49.67 \%)$. On the other hand, soaking duration means revealed that $24 \mathrm{~h}(71.11 \%)$ duration was less inhibitory than $48 \mathrm{~h}$ extracts (68.89\%) with non-significant effects (Table 11).

\section{Root elongation}

Results presented in (Table 9) demonstrated there were significant differences for varieties, concentrations and soaking duration as well as interactions between them $(\mathrm{V} \times \mathrm{Cs}, \mathrm{H} \times \mathrm{V},, \mathrm{H} \times \mathrm{Cs}$ and $\mathrm{H} \times \mathrm{V} \times \mathrm{Cs})(p \leq$ 0.05 ). Concentration means for radical length showed maximum radical length at control $(2.35 \mathrm{~mm})$, followed by $5 \mathrm{~g}(1.99 \mathrm{~mm})$ and $10 \mathrm{~g}$ $(1.79 \mathrm{~mm})$ which clearly indicated a dosedependent retardation. Maximum elongation $(3.27 \%)$ of root was observed in $P$. americanum, followed by $S$. italica $(1.01 \mathrm{~mm})$ and $L$. sativa $(0.91 \mathrm{~mm})$. The soaking duration means revealed maximum radical length in $24 \mathrm{~h}(2.17 \mathrm{~mm})$ and minimum in $48 \mathrm{~h}(1.28 \mathrm{~mm})$ (Table 12).

\section{Shoot elongation}

ANOVA for the hypocotyl length showed a significant difference for $\mathrm{V}, \mathrm{Cs}, \mathrm{H}$ and their interactive effect $\mathrm{V} \times \mathrm{Cs}, \mathrm{H} x \mathrm{~V}, \mathrm{H} \times \mathrm{Cs}$ and $\mathrm{H}$ $x \mathrm{~V} x$ Cs. $(\mathrm{p} \leq 0.05)$. However, $\mathrm{H}$ x Cs means were non-significant (Table 9). Concentration means showed that radical length was maximum in control $(2.35 \mathrm{~mm})$ that decreased significantly in a dosedependent manner to a minimum of $(1.99 \mathrm{~mm})$ at $5 \mathrm{~g}$ conc. and $10 \mathrm{~g}$ conc. $(1.79 \mathrm{~mm})$. According to varietal response, maximum radical length was noted in $P$. americanum $(3.62 \mathrm{~mm})$, followed by $S$. italica $(1.47 \mathrm{~mm})$ and $L$. sativa $(1.04 \mathrm{~mm})$. The soaking duration means revealed that $24 \mathrm{~h}(2.21 \mathrm{~mm})$ duration was more inhibitory than $48 \mathrm{~h}$ extracts $(1.87 \mathrm{~mm})$ with nonsignificant effects (Table 12).

\section{Discussion}

The present study outlined that aqueous extracts from leaves, bark, and fruits of $C$. fistula obtained by soaking for various durations invariably reduced germination and seedling growth of PA, SI and LS. It was observed that different parts of the same plant had differential toxicity not only against the different test species but also against the various growth parameters. However, the more pronounced inhibitory effect was observed in leaf extract. Our finding in this regards agrees with other researches $[11,12]$ who testified that various parts of the same plant have differential phytotoxicity. Similarly, Hussain et al. [13] reported that leaves extracts from paper mulberry were more inhibitory than other parts which are in line with our ones. The poor and delayed germination of test species under allelopathic stress might be due to either death of the embryo or the imposition of dormancy or inactivity of seeds [14].

Usually, the allelopathic effect of one plant on the other is perceived to be due to the synergetic or antagonistic effects of different compounds like alkaloids, flavonoids, phenolics, glycosides, lactones, quinines, volatile terpenes, organic acids compounds and other secondary metabolites $[15,16]$. It is suggested that in young leaves, allelopathic compounds are produced in greater quantity than mature plant leaves [17]. Our study perceived a dose-dependent inhibition of the extract from all plant parts. Though, increase in plant material (from $5 \mathrm{~g}$ to $10 \mathrm{~g}$ ) reduced the percentage germination and hypocotyl and radicle growths. This observation suggests that inhibitory effect was enhanced with increasing plant material. Our finding agrees with those of Ullah et al. [18] and Hadi et al. [19]. The present study perceived that aqueous extract from leaves, bark, and fruit obtained by soaking for various durations invariably reduced germination rate and seedling growth. The harmful effect of 
Cassia fistula parts extracts at higher concentration might be due to excess of inhibitory allelochemicals present in the extract. The presence of allelochemicals may weaken the protective ability of plant tissues against membrane lipid peroxidation and can damage the whole membrane system of plants, resulting in the ultrastructure destruction of chloroplasts, mitochondria, and nuclei. Cell destruction causes a reduction of photosynthesis and root activities. These abnormal physiological processes contribute to the inhibition of plant growth [16]. The enhanced phytotoxicity with increasing concentration and soaking duration is also noted by various workers [13, 20] thus supports our findings. Nevertheless, $48 \mathrm{~h}$ soaking as compared to $24 \mathrm{~h}$ showed less inhibition rate to germination and growth of test species in leaves extract of Cassia fistula.
This strange behavior of leaf extract was also observed by Hadi et al. [19] while working on the allelopathic impact of Phragmites karka. This reduction of inhibitory capability might be due to a degradation and/or transformation of some allelochemicals to phytotoxic compounds [21]. In a recent study, Siyar et al. [22] demonstrated that three weeds i.e., Avena fatua, Melilotus officinalis and Polypogon hissaricus had detrimental effects on germination, growth and chlorophyll concentration of wheat, which supports our results. The study is also in agreement with the findings of Muhammad et al. [10] which reported that different concentrations of leaf extracts of Ficus benjamina had negative allelopathic effects on germination and seedling growth of sunflower.

Table 9. Effects of aqueous extracts from bark of Cassia fistula on the hypocotyl length (mm) of various test plants

\begin{tabular}{|c|c|c|c|c|c|c|}
\hline \multirow{2}{*}{$\begin{array}{l}\text { Soaking } \\
\text { duration }\end{array}$} & \multirow{2}{*}{ Treat. } & \multicolumn{3}{|c|}{ Varieties } & \multirow{2}{*}{$\begin{array}{c}\text { Means } \\
\text { Hrs*Conc. }\end{array}$} & \multirow{2}{*}{$\begin{array}{c}\text { Means } \\
\text { Var.*Conc }\end{array}$} \\
\hline & & Lactuca & Setaria & Penesitum & & \\
\hline \multirow{3}{*}{$24 \mathrm{hrs}$} & Con & $1.96^{\mathrm{FG}}$ & $1.86^{\mathrm{FGH}}$ & $3.54^{\mathrm{DE}}$ & $2.45^{\mathrm{AB}}$ & $1.87^{\mathrm{D}}$ \\
\hline & $5 g$ & $1.29^{\mathrm{HIJ}}$ & $0.47^{\mathrm{KL}}$ & $4.41^{\mathrm{BC}}$ & $2.06^{\mathrm{BC}}$ & $1.29^{\mathrm{EF}}$ \\
\hline & $10 \mathrm{~g}$ & $0.69^{\mathrm{JK}}$ & $1.08^{\mathrm{IJK}}$ & $6.31^{\mathrm{A}}$ & $2.69^{\mathrm{A}}$ & $0.35^{\mathrm{G}}$ \\
\hline \multicolumn{2}{|c|}{ Means hrs*var. } & $1.31^{\mathrm{D}}$ & $1.14^{\mathrm{D}}$ & $4.7^{\mathrm{A}}$ & $2.40^{\mathrm{A}}$ & 1.85 \\
\hline \multirow{3}{*}{$48 \mathrm{hrs}$} & Con & $1.78^{\mathrm{FGH}}$ & $1.85^{\mathrm{FGH}}$ & $3.12^{\mathrm{E}}$ & $2.25^{\mathrm{BC}}$ & $0.99^{\mathrm{F}}$ \\
\hline & $5 g$ & $1.29^{\mathrm{HIJ}}$ & $1.52^{\mathrm{GHI}}$ & $4.66^{\mathrm{B}}$ & $2.49^{\mathrm{AB}}$ & $1.67^{\mathrm{DE}}$ \\
\hline & $10 \mathrm{~g}$ & $0.0^{\mathbf{L}}$ & $2.25^{\mathrm{F}}$ & $3.96^{\mathrm{CD}}$ & $2.07^{\mathrm{C}}$ & $3.33^{\mathrm{C}}$ \\
\hline \multicolumn{2}{|c|}{ Means hrs*var. } & $1.02^{\mathrm{D}}$ & $1.87^{\mathrm{C}}$ & $3.91^{\mathrm{B}}$ & $2.27^{\mathrm{B}}$ & $4.54^{\mathrm{B}}$ \\
\hline \multicolumn{2}{|c|}{ Means varieties } & $1.17^{\mathrm{C}}$ & $1.51^{\mathrm{B}}$ & $4.33^{\mathrm{A}}$ & & $5.14^{\mathrm{A}}$ \\
\hline \multicolumn{2}{|c|}{ Means concentration } & 2.35 & 2.26 & 2.38 & & \\
\hline
\end{tabular}

LSD value for Hours $=0.43$, Varieties $=0.25$, Hours*Varieties $=0.35$, Hours*Concentration $=0.35$ and Hours*Variety*Concentration $=0.61$ interactions at $\alpha 5 \%$. Mean values which are not followed by the same letter are statistically significant at $\mathrm{p}<0.05$ 
Table 10. Effects of aqueous extracts from fruits of Cassia fistula on the final germination percentage $(\%)$ of various test plants

\begin{tabular}{|c|c|c|c|c|c|c|}
\hline \multirow{2}{*}{$\begin{array}{l}\text { Soaking } \\
\text { duration }\end{array}$} & \multirow{2}{*}{ Treat. } & \multicolumn{3}{|c|}{ Varieties } & \multirow{2}{*}{$\begin{array}{c}\text { Means } \\
\text { Hrs*Conc. }\end{array}$} & \multirow{2}{*}{$\begin{array}{c}\text { Means } \\
\text { Var.*Conc. }\end{array}$} \\
\hline & & Lactuca & Setaria & Penesitum & & \\
\hline \multirow{3}{*}{$24 \mathrm{hrs}$} & Con & $60.00^{\mathbf{E F}}$ & $96.00^{\mathbf{A}}$ & $88.00^{\mathrm{ABC}}$ & $81.33^{\mathrm{A}}$ & $69.00^{\mathrm{BC}}$ \\
\hline & $5 g$ & $62.00^{\mathrm{E}}$ & $88.00^{\mathrm{ABC}}$ & $94.00^{\mathbf{A B}}$ & $81.33^{\mathrm{A}}$ & $45.00^{\mathrm{D}}$ \\
\hline & $10 \mathrm{~g}$ & $26.00^{\mathrm{H}}$ & $46.00^{\mathrm{FG}}$ & $80.00^{\mathbf{B C D}}$ & $50.67^{\mathrm{D}}$ & $35.00^{\mathrm{D}}$ \\
\hline \multicolumn{2}{|c|}{ Means hrs*var. } & 49.33 & 76.67 & 87.33 & 71.11 & $81.00^{\mathbf{A}}$ \\
\hline \multirow{3}{*}{$48 \mathrm{hrs}$} & Con & $78.00^{\text {CD }}$ & $66.00^{\mathrm{DE}}$ & $80^{\mathbf{B C D}}$ & $74.67^{\mathrm{AB}}$ & $83.00^{\mathrm{A}}$ \\
\hline & $5 \mathrm{~g}$ & $28.00^{\mathbf{E}}$ & $78.00^{\mathrm{CD}}$ & $86^{\mathrm{ABC}}$ & $64.00^{\mathrm{C}}$ & $63.00^{\mathrm{C}}$ \\
\hline & $10 \mathrm{~g}$ & $44.00^{\mathbf{G}}$ & $80.00^{\mathbf{B C D}}$ & $80^{\mathrm{BCD}}$ & $68.00^{\mathrm{D}}$ & $84.00^{\mathbf{A}}$ \\
\hline \multicolumn{2}{|c|}{ Means hrs*var. } & 50.00 & 74.67 & 82.00 & $68.89^{\mathrm{BC}}$ & $90.00^{\mathbf{A}}$ \\
\hline \multicolumn{2}{|c|}{ Means varieties } & $49.67^{\mathrm{C}}$ & $75.67^{\mathrm{B}}$ & $84.67^{\mathrm{A}}$ & & $80.00^{\mathbf{A B}}$ \\
\hline \multicolumn{2}{|c|}{ Means concentration } & $78.00^{\mathrm{A}}$ & $72.67^{\mathrm{A}}$ & $59.33^{\mathbf{B}}$ & & \\
\hline
\end{tabular}

LSD value for Varieties $=6.39$, Hours $*$ Varieties $=10.00$, Hours $*$ Concentration $=9.05$, Varieties $*$ Concentrations $=$ 11.08 and Hours*Variety*Concentration $=15.67$ interactions at $\alpha 5 \%$. Mean values which are not followed by the same letter are statistically significant at $\mathrm{p}<0.05$

Table 11. Effects of aqueous extracts from fruits of Cassia fistula on the radical length $(\mathbf{m m})$ of various test plants

\begin{tabular}{|c|c|c|c|c|c|c|}
\hline \multirow{2}{*}{$\begin{array}{l}\text { Soaking } \\
\text { duration }\end{array}$} & \multirow{2}{*}{ Treat. } & \multicolumn{3}{|c|}{ Varieties } & \multirow{2}{*}{$\begin{array}{c}\text { Means } \\
\text { Hrs*Conc. }\end{array}$} & \multirow{2}{*}{$\begin{array}{c}\text { Means } \\
\text { Var.*Conc }\end{array}$} \\
\hline & & Lactuca & Setaria & Penesitum & & \\
\hline \multirow{3}{*}{$24 \mathrm{hrs}$} & Con & $1.56^{\mathrm{CD}}$ & $1.83^{\mathrm{C}}$ & $3.29^{\mathbf{B}}$ & $2.23^{\mathrm{A}}$ & $1.54^{\mathrm{C}}$ \\
\hline & $5 \mathrm{~g}$ & $0.69^{\mathrm{DEF}}$ & $0.32^{\mathrm{F}}$ & $7.17^{\mathbf{A}}$ & $2.73^{\mathrm{A}}$ & $0.77^{\mathbf{D}}$ \\
\hline & $10 \mathrm{~g}$ & $0.17^{\mathbf{F}}$ & $1.26^{\mathrm{CDE}}$ & $3.25^{\mathrm{B}}$ & $1.56^{\mathrm{B}}$ & $0.41^{\mathbf{D}}$ \\
\hline \multicolumn{2}{|c|}{ Means hrs*var. } & $0.81^{\mathrm{C}}$ & $1.14^{\mathrm{C}}$ & $4.57^{\mathrm{A}}$ & $2.17^{\mathrm{A}}$ & $1.89^{\mathrm{C}}$ \\
\hline \multirow{3}{*}{$48 \mathrm{hrs}$} & Con & $1.52^{\mathrm{CD}}$ & $1.96^{\mathrm{C}}$ & $3.65^{\mathrm{B}}$ & $2.38^{\mathrm{A}}$ & $0.35^{\mathrm{D}}$ \\
\hline & $5 \mathrm{~g}$ & $0.84^{\mathrm{DEF}}$ & $0.37^{\mathbf{F}}$ & $1.35^{\mathrm{CDE}}$ & $0.85^{\mathrm{C}}$ & $0.79^{\mathbf{D}}$ \\
\hline & $10 \mathrm{~g}$ & $0.63^{\mathrm{EF}}$ & $0.32^{\mathrm{F}}$ & $0.91^{\text {DEF }}$ & $0.62^{\mathrm{C}}$ & $3.47^{\mathbf{B}}$ \\
\hline \multicolumn{2}{|c|}{ Means hrs*var. } & $1.00^{\mathrm{C}}$ & $0.88^{\mathrm{C}}$ & $1.97^{\mathbf{B}}$ & $1.28^{\mathrm{B}}$ & $4.26^{\mathrm{A}}$ \\
\hline \multicolumn{2}{|c|}{ Means varieties } & $0.91^{\mathbf{B}}$ & $1.01^{\mathrm{B}}$ & $3.27^{\mathrm{A}}$ & & $2.09^{\mathrm{C}}$ \\
\hline \multicolumn{2}{|c|}{ Means concentration } & $2.30^{\mathrm{A}}$ & $1.79^{\mathrm{B}}$ & $1.09^{\mathrm{C}}$ & & \\
\hline
\end{tabular}

LSD value for Hours $=10.66$, Varieties $=0.36$, Concentration $=0.36$, Hours $*$ Varieties $=0.51$, Hours $*$ Concentration $=0.51$, Varieties*Concentrations $=0.62$ and Hours*Variety*Concentration $=0.88$ interactions at $\alpha 5 \%$. Mean values which are not followed by the same letter are statistically significant at $\mathrm{p}<0.05$ 
Table 12. Effects of aqueous extracts from Fruits of Cassia fistula on the hypocotyl length (mm) of various test plants

\begin{tabular}{|c|c|c|c|c|c|c|}
\hline \multirow{2}{*}{$\begin{array}{l}\text { Soaking } \\
\text { duration }\end{array}$} & \multirow{2}{*}{ Treat. } & \multicolumn{3}{|c|}{ Varieties } & \multirow{2}{*}{$\begin{array}{c}\text { Means } \\
\text { Hrs*Conc. }\end{array}$} & \multirow{2}{*}{$\begin{array}{c}\text { Means } \\
\text { Var.*Conc }\end{array}$} \\
\hline & & Lactuca & Setaria & Penesitum & & \\
\hline \multirow{3}{*}{$24 \mathrm{hrs}$} & Con & 1.96 & 1.86 & 3.54 & 2.45 & 1.87 \\
\hline & $5 g$ & 0.87 & 0.76 & 4.64 & 2.09 & 0.82 \\
\hline & $10 \mathrm{~g}$ & 0.0 & 1.39 & 4.91 & 2.10 & 0.42 \\
\hline \multicolumn{2}{|c|}{ Means hrs*var. } & 0.94 & 1.34 & 4.36 & 2.21 & 1.85 \\
\hline \multirow{3}{*}{$48 \mathrm{hrs}$} & Con & 1.78 & 1.85 & 3.12 & 2.25 & 1.24 \\
\hline & $5 \mathrm{~g}$ & 0.77 & 1.72 & 3.17 & 1.89 & 1.32 \\
\hline & $10 \mathrm{~g}$ & 0.84 & 1.26 & 2.33 & 1.48 & 3.32 \\
\hline \multicolumn{2}{|c|}{ Means hrs*var. } & 1.13 & 1.61 & 2.87 & 1.87 & 3.90 \\
\hline \multicolumn{2}{|c|}{ Means varieties } & 1.04 & 1.47 & 3.62 & & 3.62 \\
\hline \multicolumn{2}{|c|}{ Means concentration } & 2.35 & 1.99 & 1.79 & & \\
\hline
\end{tabular}

LSD value for Hours $=0.41$, Varieties $=0.28$, Concentration $=0.28$, Hours $*$ Varieties $=0.39$, Varieties $*$ Concentrations $=0.49$ and Hours*Variety*Concentration $=0.69$ interactions at $\alpha 5 \%$. Mean values which are not followed by the same letter are statistically significant at $\mathrm{p}<0.05$

\section{Conclusion}

It was observed that extract from leaves, bark, and fruit when used as growth medium significantly reduced the germination and growth parameters of Lactuca sativa, Setaria italica and Pennisetum americanum. It is, thus, concluded that the different parts of Cassia fistula may act as natural herbicides due to the presence of allelopathic compounds. Further study is needed for confirmation and isolation of natural compound to be used as a natural herbicide.

\section{Authors' contributions}

Conceived and designed the experiments: $\mathrm{Z}$ Muhammad, Performed the experiments: WM Khan \& N Inayat, Analyzed the data: R Ullah \& A Majeed, Wrote the paper: Z Muhammad.

\section{References}

1. Cheng F \& Cheng Z (2015). Research progress on the use of plant allelopathy in agriculture and the physiological and ecological mechanisms of allelopathy. Fron Plant Sci 6: 1020.

2. Arroyo AI, Pueyo Y, Saiz H \& Alados CL (2015). Plant-plant interactions as a mechanism structuring plant diversity in a Mediterranean semi-arid ecosystem. Ecol Evol 5(22): 5305-5317.

3. Rendueles O \& Velicer GJ (2017). Evolution by flight and fight: diverse mechanisms of adaptation by actively motile microbes. The ISME J 11(2): 555.

4. Muhammad Z \& Majeed A (2014). Allelopathic effects of aqueous extracts of sunflower on wheat (Triticum aestivum L.) and maize (Zea mays L.). Pak J Bot 46(5): 1715-1718.

5. Kato-Noguchi H, Kobayashi A, Ohno O, Kimura F, Fujii Y \& Suenaga K (2014). Phytotoxic substances with allelopathic activity may be central to the strong invasive potential of Brachiaria brizantha. J Plant Physiol 171(7): 525530.

6. Latif S, Chiapusio G \& Weston LA (2017). Allelopathy and the role of allelochemicals in plant defence. In: Advances in Botanical Research, Academic Press. pp. 19-54.

7. Lindroth RL (2017). Differential toxicity of plant allelochemicals to insects: roles of enzymatic detoxication systems. In: 
Insect-Plant Interactions, CRC press. pp. $1-34$.

8. Duraipandiyan V \& Ignacimuthu $\mathrm{S}$ (2007). Antibacterial and antifungal activity of Cassia fistula L.: An ethnomedicinal plant. J Ethnopharmacol 12(3): 590-594.

9. Luximon-Ramma A, Bahorun $T$, Soobrattee MA \& Aruoma OI (2002). Antioxidant activities of phenolic, proanthocyanidin, and flavonoid components in extracts of Cassia fistula. J Agric Food Chem 50(18): 5042-5047.

10. Muhammad Z, Sobia RU \& Majeed A (2018). Allelopathic activity of leaf extracts of benjamin fig on germination and early growth potentials of sunflower. Pure Applied Biol 7(2): 486-493.

11. Iman A, Wahab S, Rastan M \& Halim M (2006). Allelopathic effect of sweet corn and vegetable soybean extracts at two growth stages on germination and seedling growth of corn and soybean varieties. J Agron 5(1): 62-68.

12. Otusanya OO, Konoh OW \& Ilori OJ (2008). Allelopathic potential of Tithonia diversifolia (Hemsl) A. Gray: Effect on germination, growth and chlorophyll accumulation of Capsicum annuum L. and Lycopersicon esculentum Mill. Inter J Bot 4: 471-475.

13. Hussain F, Niaz F, Jabeen M \& Burni T (2004). Allelopathic potential of Broussonetia papyrifera Vent. Pak J Plant Sci 10(2): 69-77.

14. Nasr M \& Shariati M (2005). The use of allelochemical to delay germination of Astragalus cycluphyllus seeds. J Agron 4: 147-150.

15. Majeed A, Chaudhry Z \& Muhammad Z (2012). Allelopathic assessment of fresh aqueous extracts of Chenopodium album L. for growth and yield of wheat (Triticum aestivum L.). Pak J Bot 44(1): 165-167.

16. Majeed A, Muhammad Z, Hussain M \& Ahmad H (2017). In vitro allelopathic effect of aqueous extracts of sugarcane on germination parameters of wheat. Acta Agric Sloven 109(2): 349-356.

17. Ahmed M \& Wardle DA (1994). Allelopathic potential of vegetative and flowering ragwort (Senecio jacobea L.) plants again associated pasture species. Plant Soil 164(1): 61-68.

18. Ullah B, Hussain F \& Ibrar M (2010). Allelopathic potential of Dodonaea viscosa (1.) Jacq. Pak J Bot 42 (4): 23832390.

19. Hadi F, Naila S, Ibrar M, Barkatullah \& Rashid A (2014). Allelopathic impact of Phragmites karka on seed germination and radical and hypocotyle growth of wheat variety sirin-2007. Sch J Agric Sci 4(7): 427-431

20. Chon SU \& Kim JD (2002). Biological activity and quantification of suspected allelochemicals from alfalfa plant parts. $J$ Agron Crop Sci 188.

21. Macías FA, Galindo JL, García-Díaz MD \& Galindo JC (2008). Allelopathic agents from aquatic ecosystems: potential biopesticides models. Phytochemistry Rev 7(1): 155-178.

22. Siyar S, Majeed A, Muhammad Z, Ali H \& Inayat N (2018). Allelopathic effect of aqueous extracts of three weed species on the growth and leaf chlorophyll content of bread wheat. Acta Ecol Sin https://doi.org/10.1016/j.chnaes.2018.05. 007. 